\title{
Mean-Variance Programming Framework in Markowitz Modeling
}

\author{
Mohamad Pazlin Bin Saion \\ Faculty of Manufacturing Technical University of Malaysia Malacca, \\ P051910004@student.utem.edu.my, manufacturing engineering field, \\ https://orcid.org/ 0000-0001-8528-5057
}

\author{
Article Info \\ Page Number: 146 - 148
}

Publication Issue:

Vol 71 No. 1 (2022)

\section{Article History}

Article Received: 18 November 2021

Revised: 03 December 2021

Accepted: 19 December 2021

Publication: 28 January 2022

\begin{abstract}
For mathematical frameworks in place, which are applied to portfolio optimization problems, the majority come in the form of risk minimization - relative to the return parameter. When it comes to the quadratic programming framework, in relation to Markowitz, its associated challenges have been countered when considerations are made to recent trends in algorithmic scholarly investigations. In particular, a linear risk function has been introduced, especially in response to the need to address problems of portfolio selection, given real constraints. In this study, the main aim is to address the problem of portfolio selection in relation to minimum transaction lots. Also, the paper focuses on an improved search model that is applied to active constrained, whereby solutions are developed for the integer programming algorithm. The proposed model is that which focuses on the relaxed problem's solution initially, before establishing solutions that are deemed closer to the continuous solutions..
\end{abstract}

Keywords: Active constrained, portfolio optimization, mixed integer programming.

\section{Introduction}

For studies that have focused on the problem of portfolio selection [1], initial insights have been gained regarding mathematical formulation. Despite the progress, drawbacks still exist when the Mean-Variance (MV) framework is considered. Particularly, the framework is associated with high computational complexity. Also, it comes with an input problem through which the parameters of $2 n+n(n-1) / 2$ are estimated. Notably, these parameters entailed the co-variances, variances, and expected returns. In finance theory, the theory has played an important role, yet its practical use is dire. Also, previous studies have strived to engage in quadratic objective function linearization [2-4].

\section{Methodology}

Currently, the MV framework comes with assets in a few thousands, and solutions have been developed to be used practically, yielding changes to model applicability. When the quadratic programming problems' 
internal point algorithm is used, there can be real-time solutions [5]. Also, the latter could be achieved via the use of linear approximations and compact factorizations [2].

On the study by Yamazaki and Konno [1], the focus was to propose an initial model for linear portfolio selection. The model's linearity was achieved through a risk function, stretching beyond the role of the classical portfolio variance. Thus, there was an absolute portfolio deviation. In the model, one of the critical features was that probabilistic assumptions were not made regarding the securities' return rates. Instead, there was a normal distribution of the return rates, based on multi-variates. With these characteristics, the model was found to be similar to Markowitz's. In another study, Kang and Zenios [3] implemented the Yamazaki and Konno framework, yielding the Mad (Mean Absolute Deviation) model. The study's aim lay in mortgage-backed securities portfolio optimization. The motivation was to illustrate the applicability of linear combination coefficients in developing a framework similar to that of Yamazaki and Konno - while ensuring that the number of constraints is halved. Similar investigations and results were documented in [4] and [5].

\section{Results and Discussion}

Indeed, most of the models of portfolio selection that have been proposed previously are those that have focused on a central assumption that a perfectly fractional state characterizes the investments, whereby for each security, real variables could aid in portfolio fraction representation. In real-world scenarios, the trading of securities comes in the form of multiples or transactions, which exist in a minimum number, yielding rounds. To ensure that the problem of portfolio selection is addressed, there is a need to develop a mixed integer programming framework relative to the rounds. When real issues are considered, the integer model's tractability is shaped by algorithm availability, especially those that are able to establish an optimal or good integer solution while achieving reasonable time of implementation. In some studies [1], the focus has been to present a general mixed integer framework. Major features that have been analyzed include minimum lost transactions' consideration, a simple heuristic, and the problem's real characteristic. Evaluations have also been conducted concerning transaction cost problems that have been fixed without and those that have been fixed with transaction lot minimization [2].

In the current study, we demonstrate that viable solution figuring comes in the form of NP-complete regardless of the nature of the risk function. The study focuses on scenarios where rounds are considered. Also, the study strives to propose a new model to solve the framework with rounds, relying on the integrizing search approach [3]. Given that the selection of securities number depends on linear or quadratic standard model of portfolio optimization and that in most cases, it is below 20, the proposed heuristics arise from a central assumption of mixed integer sub-problem solving and construction, considering the available investment choice subsets. Also, the production of the subsets is seen to rely on the relaxed problem information in terms of the reduced costs and the selected securities. The eventuality is that heuristics exhibit some degree of superiority, whereby when we consider the transaction costs, there could be the application of different mixed integer frameworks during portfolio selection. Thus, the proposed algorithms could be applied to these frameworks, allowing for easy generalization. With the focus on the utility function of the investor, the mathematical model strives to ensure risk factor tolerance reduction. Also, the role of the investment portfolio modeling aspect is seen to involve handling the Mean-VaR, especially in the absence of risk-free assets. In most cases, individual investors exhibit various utility functions and curves. These aspects are determined by the preference for investment risk attitudes. Indeed, an investor's risk- 
avoidance function could be determined based on another person's owned utility function. In this chapter, a square utility function is discussed.

\section{Conclusion}

From the suboptimal solution perspective, it was established that a specific non-basic variable $\left(\mathrm{x}_{\mathrm{N}}\right)_{\mathrm{j} *}$ could be raised to exceed the zero limit. In particular, the process could be realized by allowing the non-integer basic variable to assume integer values. For the remainder of the non-basic variable, it is allowed to remain zero. For the corresponding basic variable, integrations would be achieved by allowing a non-basic variable to play a critical role. For future works, there is a need to engage in the evaluations of the proposed heuristic algorithm's performance, especially in settings characterized by Markowitz's model variants. This area is proposed because the latter model considers rounds, even though certain issues could emerge when a quadratic model is used to provide solutions - in settings involving integer variables. Overall, this study established that the proposed model is better placed to gain application in derivative-based selection portfolio problem management. The model's superiority was confirmed in this area because it (the area) is marked by an asymmetry that poses higher risk management difficulties, confirming the model's superiority.

\section{References}

[1] Markowitz H. 1952. Portfolio Selection.Journal of Finance. 7.77-91.

[2] Sharpe W.F. 1967.A Linear Programming Approximation for A Mutual Fund Portfolio Selection, Management Science 13. 499 - 510.

[3] Sharpe W.F. 1971. A LinearProgrammingApproximation for The GeneralPortfolioAnalysisProblem. Journal of Financial and Quantitative Analysis, December. 1263 - 1275.

[4] Stone B.K. 1973. A linear programming formulation of the general portfolio selection problem. Journal of Financial and Quantitative Analysis, September. 621 - 636.

[5] Takehara H. 1991. An application of the interior point algorithm for large scale optimization in finance, Proceedings Third RAMP Symposium. Journal of the Operational Research Society of Japan, pp. 43 - 52

[6] Rajesh Kumar Chaudhary, M. K. C. (2021). The Role of School Management Towards Staff Motivation for Effective Performance in Nepal: During the Covid-19. International Journal of New Practices in Management and Engineering, 10(01), 01-11. https://doi.org/10.17762/ijnpme.v10i01.93

[7] Langaliya, V., and . . J. A. G. Gohil. "A Comparative and Comprehensive Analysis of Smart Contract Enabled Blockchain Applications: Smart Contract Enabled Blockchain Applications". International Journal on Recent and Innovation Trends in Computing and Communication, vol. 9, no. 9, Sept. 2021, pp. 16-26, doi:10.17762/ijritcc.v9i9.5489. 